\title{
Invariant structure of the hierarchy theory of fractional quantum Hall states with spin
}

\author{
M. Milovanović* and N. Read \\ Departments of Physics and Applied Physics, P.O. Box 208284 \\ Yale University, New Haven, CT 06520
}

(January 16, 2018)

\begin{abstract}
We describe the invariant structure common to abelian fractional quantum Hall effect systems with spin. It appears in a generalization of the lattice description of the polarized hierarchy that encompasses both partially polarized and unpolarized ground state systems. We formulate, using the spin-charge decomposition, conditions that should be satisfied so that the description is $\mathrm{SU}(2)$ invariant. In the case of the spin-singlet hierarchy construction, we find that there are as many $\mathrm{SU}(2)$ symmetries as there are levels in the construction. Various formalisms used before for hierarchies (field-theoretic, algebraic, and wave-functions) are also used to show the existence of a spin and charge lattice for the systems with spin. The "gluing" of the charge and spin degrees of freedom in their bulk is described by the gluing theory of lattices. The lowenergy field theories and corresponding quantum Hall lattices should serve as a starting point for the discussion of the stability of these systems.
\end{abstract}

Typeset using REVTEX

*Address after January 1: Physics Department, Technion, Haifa 32000, Israel 


\section{INTRODUCTION}

Not long after Laughlin proposed a theory [1] for the fractional quantum Hall effect (FQHE) at filling fraction $\nu=1 / q, q$ odd, the hierarchy theory [2,3] was proposed as an explanation of the occurence of the FQHE at filling fraction $p / q$ when $p$ is not 1 and $q$ is, as in the Laughlin case, an odd integer. It describes new ground states of these systems as hierarchies of Laughlin states of quasiparticles; at each level the source of the quasiparticles is the Laughlin state of the previous level. Some time later Jain proposed his construction [4] of some of these FQHE states as filled Landau levels of composite fermions, i.e. integer quantum Hall states of particles that are electrons with an even number of flux quanta attached. We can view this construction also as some kind of hierarchy and, indeed, in [5] it was proved that various constructions (the standard hierarchy that we mentioned first, the Jain construction) are different descriptions of a single underlying physical theory. This theory is described as a lattice of excitations [5], which contains all information about the quantum numbers and statistics of quasiparticles and properties of the edge states.

If we introduce an additional, spin or pseudo-spin, degree of freedom, we can construct two-component (ground) states, Halperin states [6] which are simple generalizations of the Laughlin state. Some of the Halperin states that are spin-singlets (i.e. unique states with respect to the total spin) might describe the systems for which $\vec{S}_{\text {tot }}^{2}$ and $S_{\text {tot }}^{z}$ are good quantum numbers, and Zeeman energy is small [0]. A hierarchy of these states, as an analog of the standard hierarchy in the completely spin-polarized case, was first described in [8]. From the field-theoretical point of view, in a context of a very general formulation of the FQHE with the spin degree of freedom, it was also described in [9]. On the other hand the Halperin states contain a very simple spin-charge decomposition [10], which realizes itself on the edge, that served as a starting point for a hierarchy in [11. Jain's construction of spin-singlet states [4] uses Landau levels filled with composite fermions of both spins. Naturally, this can be extended to partially polarized states with more Landau levels of one-spin particles than of the other [4, 12]. Some principles of the spin-singlet hierarchy construction were discussed in 13 also.

The goal of our paper is to show the existence of a basis-independent description for systems with the spin degree of freedom, no matter whether their ground states are partially polarized, unpolarized or spin-singlet. But, in the process of achieving this, we use the spincharge decomposition and find a structure of excitations common to all of them, which is interesting in its own right.

After a review of the Halperin states in Sec. II, in Sec. III we concentrate on the hierarchy spin-singlet systems. At the beginning, the first-level hierarchy of a general, two-component system with the (pseudo-)spin degree of freedom, is presented, based on the dual bosonic Chern-Simons field theory. Then the spin-charge decomposition is introduced, and the kind of "gluing" between the charge and spin degrees of freedom of the excitations in the bulk of these systems is described. This leads to information about the excitation lattice in this case. The lattice can be easily identified as a special case of composite lattice constructions

known in mathematics under the name "gluing theory" 14. We describe it together with a lattice, which lies in the excitation lattice, that corresponds to the order parameters of the systems. Also, in the context of the spin-charge decomposition, we use the invariance under change of hierarchy basis to formulate the spin-singlet condition. One of the bases is 
identified as the basis of Jain's construction. The most important feature of the hierarchy of spin-singlet states, which the lattices incorporate, is the existence of as many independent $\mathrm{SU}(2)$ symmetries as there are levels in the hierarchy constructions. Sec. IV and Sec. V describe, in the new formalism, a generalized spin-singlet hierarchy and the hierarchy of partially polarized states, respectively.

\section{HALPERIN STATE AND GLUING OF CHARGE AND SPIN}

An extension of the Laughlin state to two-component systems in the plane is the Halperin state [6], given by

$$
\Psi_{m m n}\left(z_{1 \uparrow} \cdots z_{N_{\uparrow}}, z_{1 \downarrow} \cdots z_{N_{\downarrow}}\right)=\prod_{i<j}\left(z_{i \uparrow}-z_{j \uparrow}\right)^{m} \prod_{k<l}\left(z_{k \downarrow}-z_{l \downarrow}\right)^{m} \prod_{r<s}\left(z_{r \uparrow}-z_{s \downarrow}\right)^{n}
$$

for two components $\uparrow$ and $\downarrow$. It is assumed that $N_{\uparrow}=N_{\downarrow}$, i.e. the state is unpolarized. It describes fermions if $m$ is an odd (positive) integer. (The exponential factors are omitted for simplicity.) In the case $m=n+1$, this state represents a ground spin-singlet state of a system with spin. If not otherwise specified, we will keep $m$ and $n$ general in the following. The pseudo-spin of two-component systems will also be called spin for short. In (2.1) we use, as is customary, the shortened form of the complete wave function, which would include spin vectors and overall antisymmetrization. The quasihole excitations carry spin $1 /[2(m-n)]$ which represents the net spin localized in the region around the quasihole. They carry $-1 /(m+n)$ (quasiholes) or $+1 /(m+n)$ (quasielectrons) unit of the electric charge. For $S_{z}=1 /[2(m-n)]$, where $S_{z}$ is the $z$ component of the quasihole spin, and in the case when $m-n=1$, a single quasihole excitation can be given by [7],

$$
\prod_{i=1}^{N_{\downarrow}}\left(z_{i \downarrow}-w\right) \Psi_{m m n},
$$

and analogously for $S_{z}=-1 /[2(m-n)]=-1 / 2$. For general $m$ and $n$, (2.2) must be generalized to the one that includes $|m-n|$ excitations.

The description of the system with each particle having a definite spin polarization can be given by an effective Chern-Simons theory [15] also, in the so- called $U(1) \times U(1)$ formulation

with two abelian gauge fields $a_{\uparrow}^{\mu}$ and $a_{\downarrow}^{\mu}[16]$. The Chern-Simons constraints that it contains are

$$
\vec{\nabla} \times \vec{a}_{\uparrow}=2 \pi\left(m \rho_{\uparrow}+n \rho_{\downarrow}\right)
$$

and

$$
\vec{\nabla} \times \vec{a}_{\downarrow}=2 \pi\left(m \rho_{\downarrow}+n \rho_{\uparrow}\right)
$$

which are simple generalizations of the constraint in the Laughlin case $(\vec{\nabla} \times \vec{a}=2 \pi m \rho)$. ( $\rho_{\uparrow}$ and $\rho_{\downarrow}$ are the densities of the up and down electrons, respectively.) The field theory can be also expressed in terms of 


$$
a_{c}^{\mu}=\frac{a_{\uparrow}^{\mu}+a_{\downarrow}^{\mu}}{2} \text { and } a_{s}^{\mu}=\frac{a_{\uparrow}^{\mu}-a_{\downarrow}^{\mu}}{2}
$$

which are charge and spin gauge fields, respectively. Their flux is connected with the charge and spin of the system as can be seen from the previous equations. The lagrangian density of the theory is then given by

$$
\begin{aligned}
\mathcal{L}= & \sum_{\sigma} i \Psi_{\sigma}^{\dagger}\left(\partial_{0}+i\left(a_{c 0}+\sigma a_{s 0}\right)-i A_{0}\right) \Psi_{\sigma}+\sum_{\sigma i} \frac{1}{2 M} \Psi_{\sigma}^{\dagger}\left(\partial_{i}+i\left(a_{c i}+\sigma a_{s i}\right)-i A_{i}\right)^{2} \Psi_{\sigma} \\
& +\frac{1}{4 \pi} \frac{2}{m+n} \epsilon^{\mu \nu \lambda} a_{c \mu} \partial_{\nu} a_{c \lambda}+\frac{1}{4 \pi} \frac{2}{m-n} \epsilon^{\mu \nu \lambda} a_{s \mu} \partial_{\nu} a_{s \lambda},
\end{aligned}
$$

where $i=x, y$ and $\Psi_{\sigma}(\sigma=+$ and - for $\uparrow$ and $\downarrow)$ is a bosonic field that represents the electron field up to the statistical transformation embodied in $\mathcal{L}$. (For simplicity we omitted the interaction and Zeeman-energy term.)

A vortex excitation of finite energy, in a charged bosonic system, can occur only if it is accompanied by an increase in the flux of the gauge fields $\left(a_{\downarrow}^{\mu}\right.$ or $\left.a_{\uparrow}^{\mu}\right)$ in the amount of an integer number of flux quanta; the flux quantum is $2 \pi$ in our units. To describe the excitations, we can integrate Eqs. (2.4) and (2.3), and relate the total changes in the flux of $a_{c}$ and $a_{s}$ fields, $\Phi_{c}$ and $\Phi_{s}$, respectively, to the total local change in the charge and spin, $Q_{c}$ and $S_{z}=Q_{s} / 2$, respectively:

$$
-Q_{c}+\frac{1}{2 \pi} \frac{2}{m+n} \Phi_{c}=0 \text { and }-Q_{s}+\frac{1}{2 \pi} \frac{2}{m-n} \Phi_{s}=0 .
$$

Taking four choices for $\left(\Phi_{c}, \Phi_{s}\right):(\pi, \pi),(\pi,-\pi),(-\pi, \pi)$, and $(-\pi,-\pi)$ gives four elementary excitations with the same quantum numbers as in the wave function approach; $\left(Q_{c}, Q_{s}\right)=$ $(1 /(m+n), 1 /(m-n)),(1 /(m+n),-1 /(m-n)),(-1 /(m+n), 1 /(m-n)),(-1 /(m+$ $n),-1 /(m-n))$, respectively. Note that the half-flux quantum changes in $\Phi_{c}$ or $\Phi_{s}$ cannot occur independently, which is an expression of the charge-spin confinement, i.e. "gluing", in the bulk of the system. Large vortices carry fluxes $(a \pi, b \pi)$ where $a+b$ is even. The Aharonov-Bohm-Berry phase $\theta$ for an interchange of two vortices, with charges and fluxes, $\left(Q_{c}^{1}, Q_{s}^{1}\right)$ and $\left(\Phi_{c}^{1}, \Phi_{s}^{1}\right)$ for vortex 1 , and $\left(Q_{c}^{2}, Q_{s}^{2}\right)$ and $\left(\Phi_{c}^{2}, \Phi_{s}^{2}\right)$ for vortex 2 , is given by

$$
\frac{\theta}{\pi}=Q_{c}^{1} \frac{\Phi_{c}^{2}}{2 \pi}+Q_{s}^{1} \frac{\Phi_{s}^{2}}{2 \pi}
$$

If we interchange two elementary vortices of the same kind the phase or statistical angle is

$$
\theta=\frac{m}{m^{2}-n^{2}} \pi
$$

We may conclude also that the excitations with fluxes $( \pm \pi(m+n), \pm \pi(m-n))$ are equivalent to one-electron excitations, which can be particles or holes of either spin, $S_{z}= \pm 1 / 2$.

Even for systems as simple as these Halperin states, we may find the lattice structure of excitations mentioned in the introduction. The excitations can be represented as vectors associated with points of a two-dimensional lattice with components being integers, $a=\Phi_{c} / \pi$ and $b=\Phi_{s} / \pi$, in a basis. The expression for the statistical angle (2.8) defines the scalar product in this lattice. For two vectors $\mathbf{v}_{1}=\left(a_{1}, b_{1}\right)$ and $\mathbf{v}_{2}=\left(a_{2}, b_{2}\right)$, it is 


$$
\mathbf{v}_{1} \cdot \mathbf{v}_{2}=\frac{a_{1} a_{2}}{2(m+n)}+\frac{b_{1} b_{2}}{2(m-n)}
$$

We see that the basis used here, $\mathbf{e}_{1}=(1,0), \mathbf{e}_{2}=(0,1)$, is orthogonal; however, these vectors, $\mathbf{e}_{1}, \mathbf{e}_{2}$, are not in the lattice as they fail to satisfy the condition $a+b=$ even. The condition expresses the fact that the excitation lattice is a special composite of two one-dimensional lattices, one for charge and one for spin. (We defer a complete description of the gluing construction to Sec. III A, and, here, we give only a simplified version of it.) The charge and spin lattice points are $(a, 0), a=0, \pm 1, \pm 2, \ldots$ and $(0, b), b=0, \pm 1, \pm 2, \ldots$, respectively and, in general, they do not belong to the excitation lattice. The gluing of the two lattices is specified by a rule that we impose on possible combinations of points from the lattices. In our case the rule is $a+b=$ even.

One way to define the charge and spin lattices is to consider the sublattice of the excitation lattice connected with the order-parameters of the system [5]. For Halperin states the order parameter excitations are specified by vectors: $\mathbf{v}=((m+n) i,(m-n) k)$ for which $i+k=$ even. The excitations represent multiples of one-electron excitations which can be particle or hole with $S_{z}= \pm 1 / 2$. The sublattice that they make can be defined as dual to the excitation lattice, i.e. the one whose vectors have integer scalar products (see Eq. (2.10) ) with all other vectors of the excitation lattice. Then, it is appropriate to define the charge and spin lattices in this sublattice as those with charge only and spin only orderparameter excitations, i.e. those defined by vectors: $\mathbf{v}_{c}=((m+n) i, 0), i=$ even, and $\mathbf{v}_{s}=(0,(m-n) k), k=$ even, respectively. Their dual lattices are the ones that we used in the preceding description of the gluing.

The Halperin states are the simplest example of the gluing construction. (A desription of the lattices specialized to the $(3,3,1)$ Halperin state was also given in Ref. [18].)

By some standard transformations, see, for example, [19], we can transform $\mathcal{L}$ into the one that describes the dual Chern-Simons theory. In the dual theory in the Laughlin case, the vortex excitations (i.e. fluxes previously) are now to be viewed as particles, and what was the particle current density becomes flux of some gauge field. In its low-energy limit the lagrangian of the dual theory in our case is

$$
\begin{aligned}
\mathcal{L}= & -\mathcal{J}_{\mu}^{s} A_{s}^{\mu}-\frac{(m-n)}{2 \pi} \epsilon^{\mu \nu \lambda} A_{s \mu} \partial_{\nu} A_{s \lambda} \\
& -\mathcal{J}_{\mu}^{c} A_{c}^{\mu}-\frac{(m+n)}{2 \pi} \epsilon^{\mu \nu \lambda} A_{c \mu} \partial_{\nu} A_{c \lambda} \\
& +\frac{1}{\pi} \epsilon^{\mu \nu \lambda} A_{\mu}^{e x t} \partial_{\nu} A_{c \lambda} .
\end{aligned}
$$

The original charge and spin current densities , $J_{\mu}^{c}$ and $J_{\mu}^{s}$, respectively, are now given by

$$
J_{c}^{\mu}=\epsilon^{\mu \nu \lambda} \frac{\partial_{\nu} A_{c \lambda}}{\pi} \text { and } J_{s}^{\mu}=\epsilon^{\mu \nu \lambda} \frac{\partial_{\nu} A_{s \lambda}}{\pi} .
$$

The currents, $\mathcal{J}_{\mu}^{c}$ and $\mathcal{J}_{\mu}^{s}$, represent the charge and spin current densities of the quasiparticles, respectively. These currents are measured in the units of charge and spin equal to the elementary quasiparticle charge and spin. To have a complete low-energy theory of the 
FQHE we must also impose the gluing among these quasiparticles, i.e. specify which fused combinations of them are allowed.

In the $\mathrm{SU}(2)$-invariant case, where $m=n+1$, this dual theory can be replaced by an explicitly $\mathrm{SU}(2)$-invariant field theory by introducing, instead of abelian gauge field $A_{s \mu}$, a nonabelian gauge field $\mathcal{A}_{\mu}=\mathcal{A}_{\mu}^{a} \tau^{a}$ where $\tau^{a}, a=1,2,3$ are Pauli matrices. Then, instead of the Chern-Simons term

$$
\frac{(m-n)}{2 \pi} \epsilon^{\mu \nu \lambda} A_{s \mu} \partial_{\nu} A_{s \lambda}
$$

with $A_{s \mu}$, in the $\mathrm{SU}(2)$-gauge-invariant theory, we have $\mathrm{SU}(2)_{k}$ Chern-Simons term where $k=1$ 20,21], i.e.

$$
\frac{k}{4 \pi} \epsilon^{\mu \nu \lambda} \operatorname{tr}\left(\mathcal{A}_{\mu} \partial_{\nu} \mathcal{A}_{\lambda}+\frac{2}{3} \mathcal{A}_{\mu} \mathcal{A}_{\nu} \mathcal{A}_{\lambda}\right)
$$

This identification of two theories is possible because $\mathrm{SU}(2)_{k=1}$ Chern-Simons theory has only excitations with abelian statistics [22] and therefore, can be formulated also in the abelian way with one abelian gauge field.

\section{SPIN-SINGLET HIERARCHY OF HALPERIN STATES AND GLUING THEORY}

\section{A. Hierarchy of Halperin states and gluing theory}

It is straightforward to derive the dual Chern-Simons theory lagrangian of the hierarchy of the Halperin states where at each level quasiparticles combine into a new Halperin state. It is a simple generalization of the lagrangian for the one-component hierarchy given in Ref. [23]. The part of the lagrangian for the first-level hierarchy that we will immediately use represents constraint conditions on (uniform) charge current densities which define the ground state. For the sake of clarity we set current densities of vortex excitations to zero. The expression for this part of the lagrangian is

$$
\begin{aligned}
\mathcal{L}= & +\epsilon_{\mu \nu \lambda} \frac{1}{2 \pi} A_{e x t}^{\mu} \partial^{\nu}\left(A_{0 \uparrow}^{\lambda}+A_{0 \downarrow}^{\lambda}\right) \\
& -\frac{m_{0}}{4 \pi} \epsilon_{\mu \nu \lambda} A_{0 \uparrow}^{\mu} \partial^{\nu} A_{0 \uparrow}^{\lambda}-\frac{m_{0}}{4 \pi} \epsilon_{\mu \nu \lambda} A_{0 \downarrow}^{\mu} \partial^{\nu} A_{0 \downarrow}^{\lambda}-\frac{n_{0}}{2 \pi} \epsilon_{\mu \nu \lambda} A_{0 \uparrow}^{\mu} \partial^{\nu} A_{0 \downarrow}^{\lambda}-\frac{n_{0}}{2 \pi} \epsilon_{\mu \nu \lambda} A_{0 \downarrow}^{\mu} \partial^{\nu} A_{0 \uparrow}^{\lambda} \\
& -\frac{1}{2 \pi} \epsilon_{\mu \nu \lambda} A_{0 \uparrow}^{\mu} \partial^{\nu} A_{1 \downarrow}^{\lambda}-\frac{1}{2 \pi} \epsilon_{\mu \nu \lambda} A_{0 \downarrow}^{\mu} \partial^{\nu} A_{1 \uparrow}^{\lambda} \\
& -\frac{m_{1}}{4 \pi} \epsilon_{\mu \nu \lambda} A_{1 \uparrow}^{\mu} \partial^{\nu} A_{1 \uparrow}^{\lambda}-\frac{m_{1}}{4 \pi} \epsilon_{\mu \nu \lambda} A_{1 \downarrow}^{\mu} \partial^{\nu} A_{1 \downarrow}^{\lambda}-\frac{n_{1}}{2 \pi} \epsilon_{\mu \nu \lambda} A_{1 \uparrow}^{\mu} \partial^{\nu} A_{1 \downarrow}^{\lambda}-\frac{n_{1}}{2 \pi} \epsilon_{\mu \nu \lambda} A_{1 \downarrow}^{\mu} \partial^{\nu} A_{1 \uparrow}^{\lambda},
\end{aligned}
$$

where $A_{\text {ext }}^{\mu}$ represents an external electromagnetic field, numbers 0 and 1 denote the levels

of the hierarchy, and the previous $m$ and $n$ are now $m_{0}$ and $n_{0}$. The equations of motion obtained from (3.1) are

$$
\begin{gathered}
N_{\phi}=m_{0} N_{\sigma}^{0}+n_{0} N_{-\sigma}^{0}+\alpha N_{-\sigma}^{1}, \\
0=N_{\sigma}^{0}+m_{1} \alpha N_{\sigma}^{1}+n_{1} \alpha N_{-\sigma}^{1}
\end{gathered}
$$


with $\sigma=\uparrow$ or $\downarrow, N_{\sigma}^{0}$ and $N_{\sigma}^{1}$ denote the total numbers of electrons and quasiparticles, respectively, $\alpha=+1$ for quasiholes or $\alpha=-1$ for quasielectrons, and $N_{\phi}$ is the number of flux quanta through the system. $m_{1}$ is an even integer because we consider the quasiparticles as bosons.

By defining new gauge fields, analogously to what we did for a single Halperin state (2.5), the lagrangian density (3.1) can be rewritten in a form with charge and spin variables only,

$$
\begin{aligned}
\mathcal{L}= & -\frac{\left(m_{0}-n_{0}\right)}{2 \pi} \epsilon_{\mu \nu \lambda} A_{s 0}^{\mu} \partial^{\nu} A_{s 0}^{\lambda}-\frac{\left(m_{0}+n_{0}\right)}{2 \pi} \epsilon_{\mu \nu \lambda} A_{c 0}^{\mu} \partial^{\nu} A_{c 0}^{\lambda}+\epsilon_{\mu \nu \lambda} \frac{A_{e x t}^{\mu} \partial^{\nu} A_{c 0}^{\lambda}}{\pi} \\
& -\frac{\left(m_{1}-n_{1}\right)}{2 \pi} \epsilon_{\mu \nu \lambda} A_{s 1}^{\mu} \partial^{\nu} A_{s 1}^{\lambda}-\frac{\left(m_{1}+n_{1}\right)}{2 \pi} \epsilon_{\mu \nu \lambda} A_{c 1}^{\mu} \partial^{\nu} A_{c 1}^{\lambda} \\
& -\epsilon_{\mu \nu \lambda} \frac{a_{c 0}^{\mu} \partial^{\nu} A_{c 1}^{\lambda}}{\pi}-\epsilon_{\mu \nu \lambda} \frac{A_{s 0}^{\mu} \partial^{\nu} A_{s 1}^{\lambda}}{\pi}-\mathcal{J}_{\mu}^{c 0} A_{c 0}^{\mu}-\mathcal{J}_{\mu}^{s 0} A_{s 0}^{\mu}-\mathcal{J}_{\mu}^{c 1} A_{c 1}^{\mu}-\mathcal{J}_{\mu}^{s 1} A_{s 1}^{\mu},
\end{aligned}
$$

where we included also the vortex excitations with respect to 0 and 1 level described by current densities $\mathcal{J}_{\mu}^{c 0}$ and $\mathcal{J}_{\mu}^{s 0}$, and $\mathcal{J}_{\mu}^{c 1}$ and $\mathcal{J}_{\mu}^{s 1}$, respectively. As before the current densities are measured in the units of the corresponding quasiparticle charge and spin. The form of the lagrangian in (3.3) suggests the definition of two matrices, charge

$$
\Lambda_{c}=\left(\begin{array}{cc}
m_{0}+n_{0} & 1 \\
1 & m_{1}+n_{1}
\end{array}\right)
$$

and spin

$$
\Lambda_{s}=\left(\begin{array}{cc}
m_{0}-n_{0} & 1 \\
1 & m_{1}-n_{1}
\end{array}\right)
$$

They are analogous to the one-dimensional ones, $m_{0}+n_{0}$ and $m_{0}-n_{0}$, for the Halperin state in Sec. II.

The elementary excitations from each level of the constructed hierarchy are of the type discussed in the section on the Halperin state. Therefore, if we introduce vectors

$$
N^{c}=\left(\begin{array}{c}
N_{0}^{c} \\
N_{1}^{c}
\end{array}\right) \text { and } N^{s}=\left(\begin{array}{c}
N_{0}^{s} \\
N_{1}^{s}
\end{array}\right)
$$

with entries denoting how many, with respect to the level 0 or 1 , quasiparticles that carry only charge $(c)$ or only spin $(s)$ are created, not any pair of these vectors represents a system excitation. (The entries can be negative denoting the number of quasiparticles with opposite charge or spin.) Because of the gluing of these particles in the bulk of the system, only integral linear combinations of the following elementary excitations from the zeroth level of the hierarchy:

$$
\left\{\left(\begin{array}{l}
1 \\
0
\end{array}\right)_{c},\left(\begin{array}{l}
1 \\
0
\end{array}\right)_{s}\right\} \text { and }\left\{\left(\begin{array}{l}
1 \\
0
\end{array}\right)_{c},\left(\begin{array}{c}
-1 \\
0
\end{array}\right)_{s}\right\},
$$

and 


$$
\left\{\left(\begin{array}{l}
0 \\
1
\end{array}\right)_{c},\left(\begin{array}{l}
0 \\
1
\end{array}\right)_{s}\right\} \text { and }\left\{\left(\begin{array}{l}
0 \\
1
\end{array}\right)_{c},\left(\begin{array}{c}
0 \\
-1
\end{array}\right)_{s}\right\}
$$

from the first level of the hierarchy, are allowed. If we denote by $x$ a vector from the charge part and by $y$ a vector from the spin part, the operations allowed on the vectors in (3.7) and $(3.8)$ are

$$
\text { 1. }\left\{x_{1}, y_{1}\right\}+\left\{x_{2}, y_{2}\right\} \stackrel{\text { def }}{=}\left\{x_{1}+x_{2}, y_{1}+y_{2}\right\}
$$

and if $\alpha$ is an integer

$$
\text { 2. } \alpha\{x, y\} \stackrel{\text { def }}{=}\{\alpha x, \alpha y\} \text {. }
$$

These are familiar operations for a direct sum of two vector spaces. In addition, if the scalar product is defined by

$$
\left\{x_{1}, y_{1}\right\} \cdot\left\{x_{2}, y_{2}\right\}=x_{1} \cdot x_{2}+y_{1} \cdot y_{2}
$$

the direct sum is orthogonal.

To any excitation we can assign the corresponding change in fluxes in the dual ChernSimons theory (3.3). Then the Aharonov-Bohm-Berry phase for an interchange of two identical excitations gives the statistical angle for that excitation and it is equal to

$$
\frac{\theta}{\pi}=N^{c} \frac{\Lambda_{c}^{-1} N^{c}}{2}+N^{s} \frac{\Lambda_{c}^{-1} N^{s}}{2}
$$

The charge density in the dual Chern-Simons theory (3.3) can be read off as a coefficient in front of $A_{\mu}^{e x t}$ and is equal to

$$
\rho=\frac{1}{\pi} \epsilon_{\mu \nu \lambda} \partial^{\nu} A_{0 c}^{\lambda}
$$

(As before in the theory a unit of charge corresponds to a half of the flux quantum.) Therefore the charge of the excitation is given by

$$
Q_{c}=\left(\Lambda_{c}^{-1}\right)_{0 I} N_{I}^{c}
$$

and analogously the spin by

$$
S_{z}=\left(\Lambda_{s}^{-1}\right)_{0 I} N_{I}^{s} \frac{1}{2}
$$

We can rewrite the coupling between the charge density and external field [25] as

$$
\rho A^{e x t}=\frac{1}{\pi} \epsilon_{\mu \nu \lambda} A_{e x t}^{\mu} \partial^{\nu} A_{0 c}^{\lambda}=\frac{1}{\pi} \epsilon_{\mu \nu \lambda} A_{e x t}^{\mu} \sum_{i=0,1} V_{i} \partial^{\nu} A_{i c}^{\lambda},
$$

where 


$$
V=\left(\begin{array}{l}
1 \\
0
\end{array}\right) .
$$

Along with the $S$ transformations the vector $V$ will change (to leave the coupling invariant and characterize the nature of the coupling between the external field $A_{\mu}^{\text {ext }}$ and internal gauge fields in a new basis). Therefore, we may rewrite the physical quantity $Q_{c}$ in a basis-independent way as

$$
Q_{c}=V \Lambda_{c}^{-1} N^{c}
$$

and similar arguments lead to

$$
S_{z}=V \Lambda_{s}^{-1} N^{s} \frac{1}{2}
$$

The given description of the excitations may lead us to the conclusion that they are best described by an orthogonal direct sum of two two-dimensional lattices, one for spin $L_{s}^{*}$ and one for charge $L_{c}^{*}$. With fixed bases in them, $\left\{e_{\alpha}^{c} ; \alpha=0,1\right\}$ and $\left\{e_{\beta}^{s} ; \beta=0,1\right\}$, the excitations are given by the integer vectors (3.6), and their scalar products are defined on these lattices with two Gram matrices, $\left(\Lambda_{c}^{-1} / 2\right)_{\alpha \beta}=e_{\alpha}^{c} \cdot e_{\beta}^{c}$ and $\left(\Lambda_{s}^{-1} / 2\right)_{\alpha \beta}=e_{\alpha}^{s} \cdot e_{\beta}^{s}$. The Gram matrices are read off from the expression (3.12) for the statistical angle, which plays the role of the scalar product for the vectors (3.6). But, as in the case of the systems with the Halperin ground states, the excitation lattice is the result of the gluing construction, and it is a sublattice of this direct sum. Because of the requirement for the flux quantization, only vectors (3.6) whose components satisfy conditions,

$$
N_{0}^{c} \pm N_{0}^{s}=\text { even and } N_{1}^{c} \pm N_{1}^{s}=\text { even }
$$

belong to the excitation lattice.

The order-parameter lattice is a sublattice of the excitation lattice, and can be defined in the following way. (Note that we use the words the order-parameter lattice instead of the condensate lattice of Ref. [5].) If, in the standard-hierarchy basis, we define the vector $W$,

$$
W=\left(\begin{array}{l}
0 \\
1
\end{array}\right)
$$

the numbers

$$
Q^{q p}=W \Lambda_{c}^{-1} N^{c} \text { and } S_{z}^{q p}=\left(W \Lambda_{s}^{-1} N^{s}\right) / 2,
$$

have the meaning of the quasiparticle charge and spin. Then the order-parameter lattice consists of points whose vectors $N^{c}$ and $N^{s}$ satisfy conditions $Q \pm 2 S_{z}=$ even and $Q^{q p} \pm 2 S_{z}^{q p}=$ even (where $Q, 2 S_{z}, Q^{p q}$, and $2 S_{z}^{q p}$ are integers). Using this definition and the definition of the scalar product (3.12), we can see that the dual of the order-parameter lattice is the excitation lattice. Namely, all vectors $N^{c}$ and $N^{s}$, which have integer scalar products with all vectors of the order-parameter lattice, satisfy the conditions (3.20).

Two sublattices can be defined in the order-parameter lattice: the charge lattice $L_{c}$, for which $Q=$ even, $2 S_{z}=0, Q^{q p}=$ even, and $2 S_{z}=0$, and the spin lattice $L_{s}$, for which 
$Q=0,2 S_{z}=$ even, $Q^{q p}=0$, and $2 S_{z}=$ even. The dual charge lattice $L_{c}^{*}$ consists of arbitrary integer-component $N^{c}$ vectors, and $N^{s}=\left(\begin{array}{l}0 \\ 0\end{array}\right)$, and the dual spin lattice $L_{s}^{*}$ has $N^{c}=\left(\begin{array}{l}0 \\ 0\end{array}\right)$ and arbitrary integer-component $N^{s}$ vectors. We used these two lattices, $L_{c}^{*}$ and $L_{s}^{*}$, to describe the gluing construction. Because their Gram matrices are $\Lambda_{c}^{-1} / 2$ and $\Lambda_{s}^{-1} / 2$, the Gram matrix for the charge lattice $L_{c}$ is $2 \Lambda_{c}$, and for the spin lattice $L_{s}$ is $2 \Lambda_{s}$ [14.

The gluing construction of lattices can be defined in a basis-independent way on a small number of vectors. In the following we will define [14] such a construction for an integral lattice i.e. the one with an integer scalar product or integer Gram matrix [14]. The lattice $L$ constructed by the gluing theory contains a sublattice which is a direct sum

$$
L_{1} \oplus L_{2}
$$

of two integral sublattices $L_{1}$ and $L_{2}$. Any vector of $L$ can be written as

$$
\mathbf{y}=\mathbf{y}_{1} \oplus \mathbf{y}_{2},
$$

where each component $\mathbf{y}_{i}$ belongs to $L_{i}^{*}$, the dual lattice of $L_{i}$. Therefore, $\mathbf{y}_{i}$ is not necessarily in $L_{i}$. To classify candidates for $\mathbf{y}_{i}$ we may consider the sets obtained by adding to each $\mathbf{y}_{i}$ all vectors from $L_{i}$. Then $\mathbf{y}_{i}$ in (3.24) are representatives of the cosets of $L_{i}$ in $L_{i}^{*}$. $\mathbf{y}^{\prime}$ s must have integer scalar products with one another, and are closed under addition modulo $L_{1}+L_{2}$. They are known under the name glue vectors.

We may identify our order-parameter lattice construction, with the basis independent, abstract notion of the gluing of two integral lattices $L_{c}$ and $L_{s}$ (which play roles of $L_{1}$ and $L_{2}$ in the preceding paragraph). As we already said, in the standard-hierarchy basis the matrices $2 \Lambda_{c}$ and $2 \Lambda_{s}$ (see Eq. (3.4) and (3.5)) are the Gram matrices of the two integral lattices $L_{c}$ and $L_{s}$. If $\mathbf{a}_{1 c}$ and $\mathbf{a}_{2 c}$ denote the standard-hierarchy basis vectors of the lattice $L_{c}$, the matrix $2 \Lambda_{c}$ encodes information about their scalar products in the following way [14]:

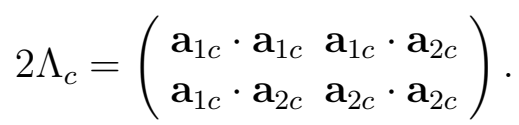

Therefore, if $\mathbf{e}_{1}=(1,0)$ and $\mathbf{e}_{2}=(0,1)$ are the orthogonal basis vectors with unit norms, the vectors $\mathbf{a}_{1 c}$ and $\mathbf{a}_{2 c}$ can be expressed as

$$
\mathbf{a}_{1 c}=\sqrt{2\left(m_{0}+n_{0}\right)} \mathbf{e}_{1},
$$

and

$$
\mathbf{a}_{2 c}=\sqrt{\frac{2}{m_{0}+n_{0}}} \mathbf{e}_{1}+\sqrt{\frac{2 D_{c}}{m_{0}+n_{0}}} \mathbf{e}_{2},
$$

where $D_{c}=\operatorname{det} \Lambda_{c}$. The dual (excitation)-lattice basis vectors are 


$$
\mathbf{b}_{1 c}=\sqrt{\frac{1}{2\left(m_{0}+n_{0}\right)}} \mathbf{e}_{1}-\sqrt{\frac{1}{2 D_{c}\left(m_{0}+n_{0}\right)}} \mathbf{e}_{2}
$$

and

$$
\mathbf{b}_{2 c}=\sqrt{\frac{\left(m_{0}+n_{0}\right)}{2 D_{c}}} \mathbf{e}_{2} .
$$

(They are obtained from the requirement that any dual-lattice vector $\mathbf{v}=p \mathbf{e}_{1}+q \mathbf{e}_{2}, p$ and $q$, in general, rational, has an integral scalar product with vectors $\mathbf{w}=k \mathbf{a}_{1 c}+l \mathbf{a}_{2 c}, k$ and $l$ integer, of the integral lattice $L_{c}$.) We may check that the volume of the elementary integral-lattice $\left(L_{c}\right)$ cell is $4 D_{c}=\operatorname{det}\left\{2 \Lambda_{c}\right\}$ times larger than the volume of the elementary dual lattice cell, i.e.

$$
\frac{\left|\mathbf{a}_{1 c} \times \mathbf{a}_{2 c}\right|}{\left|\mathbf{b}_{1 c} \times \mathbf{b}_{2 c}\right|}=4 D_{c} .
$$

This is a one way to see that there are $\operatorname{det}\left\{2 \Lambda_{c}\right\}$, i.e. the determinant of the integral-lattice Gram matrix, coset representatives of $L_{c}$ in $L_{c}^{*}$ [14. To find a complete set of them, we require that

$$
0 \leq \mathbf{v} \cdot \mathbf{e}_{1}<\mathbf{a}_{1} \cdot \mathbf{e}_{1} \text { and } 0 \leq \mathbf{v} \cdot \mathbf{e}_{2}<\mathbf{a}_{2} \cdot \mathbf{e}_{2}
$$

that is we look for all that are in the volume of the integral-lattice $\left(L_{c}\right)$ elementary cell. To give an example, we specialize to the case for which $m_{0}+n_{0}=1$. Taking the conditions (3.31) into account, we get

$$
\left(\begin{array}{l}
0 \\
0
\end{array}\right)_{c},\left(\begin{array}{l}
0 \\
1
\end{array}\right)_{c}, \ldots,\left(\begin{array}{c}
0 \\
2\left(m_{1}+n_{1}\right)-3
\end{array}\right)_{c},
$$

and

$$
\left(\begin{array}{l}
1 \\
1
\end{array}\right)_{c},\left(\begin{array}{l}
1 \\
2
\end{array}\right)_{c}, \ldots,\left(\begin{array}{c}
1 \\
2\left(m_{1}+n_{1}\right)-2
\end{array}\right)_{c}
$$

as a set of the coset representatives of $L_{c}$ in $L_{c}^{*}$. (There are $4\left[\left(m_{1}+n_{1}\right)-1\right]=4 D_{c}$ of them.) The components of the vectors are defined relative to the dual-lattice $\left(L_{c}^{*}\right)$ basis vectors $\left(\mathbf{b}_{1 c}\right.$ and $\mathbf{b}_{2 c}$ ). The analysis for the spin lattices can be repeated in a similar way and the results only differ from the charge case in that, that we have to change the sign of $n_{i}, i=0,1$. For this special case $\left(m_{0} \pm n_{0}=1\right)$ the glue vectors from the definition of the gluing construction are

$$
\begin{gathered}
\left(\begin{array}{l}
0 \\
0
\end{array}\right)_{c} \oplus\left(\begin{array}{l}
0 \\
0
\end{array}\right)_{s},\left(\begin{array}{l}
1 \\
1
\end{array}\right)_{c} \oplus\left(\begin{array}{l}
1 \\
1
\end{array}\right)_{s} \\
\left(\begin{array}{c}
0 \\
\left(m_{1}+n_{1}\right)-1
\end{array}\right)_{c} \oplus\left(\begin{array}{c}
0 \\
\left(m_{1}-n_{1}\right)-1
\end{array}\right)_{s} \text { and }\left(\begin{array}{c}
1 \\
m_{1}+n_{1}
\end{array}\right)_{c} \oplus\left(\begin{array}{c}
1 \\
m_{1}-n_{1}
\end{array}\right)_{s},
\end{gathered}
$$

where we also assumed that $m_{1}+n_{1}$ is an even number. The last assumption is appropriate, as we will see, in the case of the spin-singlet constructions. The glue vectors satisfy the 
gluing rule that we found considering the elementary excitations of the first-level hierarchy; we glue only those coset-representative vectors for which components for each hierarchy level (in the standard-hierarchy basis) are both even or both odd integers. They have integral norms, integral scalar products with one another, and are closed under addition modulo $L_{c} \oplus L_{s}$. All other vectors of the order-parameter lattice $L$ are obtained by adding the ones from $L_{c} \oplus L_{s}$ to these glue vectors. From the order-parameter lattice the excitation lattice can be constructed, being dual of the order-parameter lattice.

\section{B. Spin-singlet condition and first-level spin-singlet hierarchy}

The spin-singlet states of the general two-component hierarchy should have an explicitly $\mathrm{SU}(2)$-invariant field-theoretical description. So in that case, it should be possible to cast the low-energy lagrangian density in (3.3) in an explicitly SU(2)-invariant form. The low-energy form in (3.3) contains a possibility to make transformations

$$
\begin{gathered}
\Lambda_{c}^{\prime}=S_{c}^{\top} \Lambda_{c} S_{c} \text { and } \Lambda_{s}^{\prime}=S_{s}^{\top} \Lambda_{s} S_{s}, \\
\mathcal{J}_{c}^{\prime}=S_{c} \mathcal{J}_{c} \text { and } \mathcal{J}_{s}^{\prime}=S_{s} \mathcal{J}_{s},
\end{gathered}
$$

and simultaneous inverse transformations on the gauge fields

$$
S_{c}^{-1} A_{c}=A_{c}^{\prime} \text { and } S_{s}^{-1} A_{s}=A_{s}^{\prime}
$$

as found in the polarized case in [5.25.26]. The matrices $S_{c}$ and $S_{s}$ must be integer matrices, $\left|\operatorname{det} S_{c}\right|=1$, and $\left|\operatorname{det} S_{s}\right|=1$, because they must map the excitation vectors with integer components (3.6) into vectors with integer components in a one-to-one fashion [5, 25, 26]. The form of the $\mathrm{SU}(2)_{k=1}$ Chern-Simons term in (2.14) suggests that, only when $\Lambda_{s}^{\prime}$ is in a diagonal form, we would be able to cast the theory in a $\mathrm{SU}(2)$-explicitly-invariant form. Therefore, for any spin-singlet construction, we must have this possibility of having a transformation $S_{s}$ which converts $\Lambda_{s}$ in a diagonal form. Moreover, repeating the replacement of (2.13) with (2.14) in this case, we require that the diagonal entries of the transformed $\Lambda_{s}$ must be 1 or -1 , i.e. that we have a theory with as many $\mathrm{SU}(2)_{k=1}$ Chern-Simons terms as the number of the levels in the hierarchy (including the zeroth one). Only with this requirement the $\mathrm{SU}(2)$-invariant theory has abelian statistics, which must be the statistics of the excitations in any hierarchy built up from the Halperin states.

The formulated spin-singlet condition implies that the spin lattice $L_{s}$ and the dual spin lattice $L_{s}^{*}$, in the spin-singlet hierarchy, should be of the simple square-lattice kind. This is implied because of the existence of the basis with the diagonal form of $\Lambda_{s}$ where the absolute values of the diagonal matrix are the same. Comparing the Gram matrix $2 \Lambda_{s}$ for the spin lattice $L_{s}$ and the Gram matrix $(1 / 2) \Lambda_{s}^{-1}$ for the dual spin lattice $L_{s}^{*}$, we may conclude that $L_{s}$ is a square lattice with the basis vectors of twice the length of those describing $L_{s}^{*}$.

In the following, the condition formulated in the first paragraph of this subsection will be applied to the construction of the first-level spin-singlet hierarchy. We will find all two-bytwo spin matrices of the form (3.5) with $m_{1}$ even, for which there exist the integer matrices $S_{s}$ (with $\left|\operatorname{det} S_{s}\right|=1$ ) that transform them into the diagonal form with -1 or 1 on the diagonal. 
The same transformation that is applied to the spin part will be applied to the charge part $\left(S_{s}=S_{c}=S\right)$, and we will identify the new basis as the basis of Jain's construction.

We generate our spin-singlet hierarchy by successively applying the spin-singlet condition on each level of the hierarchy. Therefore, the two diagonal matrices that we should consider in the spin part, $D_{s}^{i}, i=1,2\left(D_{s}^{i}=S^{i \top} \Lambda_{s}^{i} S^{i}\right)$, are

$$
D_{s}^{1}=\left(\begin{array}{cc}
1 & 0 \\
0 & -1
\end{array}\right) \quad \text { and } \quad D_{s}^{2}=\left(\begin{array}{ll}
1 & 0 \\
0 & 1
\end{array}\right)
$$

the first diagonal entry is the condition $m_{0}-n_{0}=1$ on the zeroth level of the hierarchy. (We do not consider the $m_{0}-n_{0}=-1$ condition for a reversed magnetic field because the corresponding cases reduce to the ones given by (3.38)).

The first case, because $\operatorname{det} \Lambda_{s}=\operatorname{det} D_{s}$, corresponds to the condition $m_{1}-n_{1}=0$. The transformation matrix $S_{c}=S_{s}=S$ in (3.35) in this case is

$$
S^{1}=\left(\begin{array}{cc}
1 & 1 \\
0 & -1
\end{array}\right)
$$

and the charge part in the new basis is

$$
D_{c}^{1}=\left(\begin{array}{cc}
m_{0}+n_{0} & m_{0}+n_{0}-1 \\
m_{0}+n_{0}-1 & \left(m_{0}+n_{0}\right)+\left(m_{1}+n_{1}\right)-2
\end{array}\right) .
$$

( $D_{s}^{1}$ has all $n$ 's with minus sign.)

To understand the physical meaning of the new basis we specialize to the case $m_{0}+n_{0}=1$, that is the case where the zeroth level is the completely filled lowest Landau level of both spins. Then the charge and spin matrices are

$$
D_{c}^{1}=\left(\begin{array}{cc}
1 & 0 \\
0 & \left(m_{1}+n_{1}\right)-1
\end{array}\right) \quad \text { and } \quad D_{s}^{1}=\left(\begin{array}{cc}
1 & 0 \\
0 & -1
\end{array}\right) .
$$

The latter means that there are two independent spin-singlet systems. If, in addition, both $m_{1}$ and $n_{1}$ are chosen to be nonpositive even numbers, i.e. $m_{1}=n_{1}=-2 n$ where $n=1,2, \ldots$, the second system is described by a Halperin state in a reverse magnetic field. The filling fraction is $2+2 /\left[\left(m_{1}+n_{1}\right)-1\right]=2-2 /(4 n+1)$. The form of the description, given by (3.41) of these Halperin states of holes, suggests that we are in the basis of Jain's construction. Indeed, the form of the general hierarchy construction, represented by the matrix $D_{c}^{1}$ in Eq. (3.40), tells us that it is the result of the two operations which characterize Jain's construction [4,5,25]; first combining with one Landau level (exemplified in (3.41)) and then attaching flux to electrons to get composite fermions. Explicitly, it is

$$
D_{c}^{1}=\left(\begin{array}{cc}
1 & 0 \\
0 & \left(m_{1}+n_{1}\right)-1
\end{array}\right)+\left(m_{0}+n_{0}-1\right)\left(\begin{array}{ll}
1 & 1 \\
1 & 1
\end{array}\right)
$$

with the pseudo-identity matrix (with all entries equal to one), which always comes with the spin-independent flux-attaching operation. The number of the flux quanta attached is $\left(m_{0}+n_{0}-1\right) / 2$, which is an integer. (Always, to get physical quantities that are measured 
in the unit of one flux quantum, we have to divide the defined matrices of the theory by two because they describe a theory where the unit for flux is one half of the flux quantum.)

Having identified which values of $m_{1}$ and $n_{1}$ give spin-singlets and recognized that, in the case $m_{1}=n_{1}=-2 n$, in the standard-hierarchy picture we have a quasihole system, we are ready to construct the corresponding wave function in the fractional-statistics representation [27] for this case. If we use numbers $p$ and $q$ to describe the statistical angles of the quasiholes of the zeroth level as (see Eq. (2.8) and (2.9))

$$
\theta_{i}=\frac{m_{0}}{m_{0}^{2}-n_{0}^{2}} \pi=p \pi
$$

for the exchange of the same-spin quasiholes, and

$$
\theta_{d}=-\frac{n_{0}}{m_{0}^{2}-n_{0}^{2}} \pi=q \pi
$$

for the exchange of the opposite-spin quasiholes, the wave function is of the form [27]

$$
\begin{aligned}
\Psi\left(z_{1 \uparrow}, \ldots, z_{N \downarrow}\right)= & \exp \left\{-\frac{1}{4} \sum\left|z_{i}\right|^{2}\right\} \prod_{i<j}\left(z_{i \uparrow}-z_{j \uparrow}\right)^{m_{0}} \prod_{i<j}\left(z_{i \downarrow}-z_{j \downarrow}\right)^{m_{0}} \prod_{i, j}\left(z_{i \downarrow}-z_{j \uparrow}\right)^{n_{0}} \\
& \times \int d^{2} w_{1 \uparrow} \cdots \int d^{2} w_{M \downarrow} \prod_{i, j}\left(z_{i \uparrow}-w_{j \downarrow}\right) \prod_{i, j}\left(z_{i \downarrow}-w_{j \uparrow}\right) \\
& \times \prod_{i<j}\left|w_{i \uparrow}-w_{j \uparrow}\right|^{2 p} \prod_{i<j}\left|w_{i \downarrow}-w_{j \downarrow}\right|^{2 p} \prod_{i, j}\left|w_{i \uparrow}-w_{j \downarrow}\right|^{2 q} \\
& \times \prod_{i<j}\left(\bar{w}_{i \uparrow}-\bar{w}_{j \uparrow}\right)^{2 n} \prod_{i<j}\left(\bar{w}_{i \downarrow}-\bar{w}_{j \downarrow}\right)^{2 n} \prod_{i, j}\left(\bar{w}_{i \downarrow}-\bar{w}_{j \uparrow}\right)^{2 n} \exp \left\{-\frac{1}{2\left(m_{0}+n_{0}\right)} \sum\left|w_{i}\right|^{2}\right\}
\end{aligned}
$$

The pseudo-wave function [27], which incorporates the effects of fractional statistics and describes the physics of the system solely in terms of the quasiparticle coordinates, is

$$
\Psi\left(\bar{w}_{1 \uparrow}, \ldots, \bar{w}_{M \downarrow}\right)=\prod_{i<j}\left(\bar{w}_{i \downarrow}-\bar{w}_{j \downarrow}\right)^{p+2 n} \prod_{i<j}\left(\bar{w}_{i \uparrow}-\bar{w}_{j \uparrow}\right)^{p+2 n} \prod_{i<j}\left(\bar{w}_{i \downarrow}-\bar{w}_{j \uparrow}\right)^{q+2 n} .
$$

The condition [28] for spin-singlet states for quasiparticles with fractional statistics [22] is

$$
\left(\sum_{j=1}^{M / 2} e(i,[j]) \exp \left\{-i \theta_{s}\right\}+1\right) \Psi\left(\bar{w}_{1 \uparrow}, \ldots, \bar{w}_{M \downarrow}\right)=0,
$$

where $e(i,[j])$ denotes an exchange between two particles with opposite spin. Because $p-q=$ 1 , we can use a decomposition of the wave function in the form

$$
\begin{aligned}
\Psi\left(\bar{w}_{1 \uparrow}, \ldots, \bar{w}_{M \downarrow}\right)= & \prod_{i<j}\left(\bar{w}_{i \downarrow}-\bar{w}_{j \downarrow}\right)^{p+2 n-1} \prod_{i<j}\left(\bar{w}_{i \uparrow}-\bar{w}_{j \uparrow}\right)^{p+2 n-1} \prod_{i<j}\left(\bar{w}_{i \downarrow}-\bar{w}_{j \uparrow}\right)^{p+2 n-1} \\
& \times \prod_{i<j}\left(\bar{w}_{i \uparrow}-\bar{w}_{j \uparrow}\right) \prod_{i<j}\left(\bar{w}_{i \downarrow}-\bar{w}_{j \downarrow}\right)
\end{aligned}
$$

where a spin-independent factor multiplies the filled lowest Landau level of both spins which is a spin-singlet, and see that it obeys the spin-singlet condition. This coincides with our 
intuitive expectation that, in the case of the spin-singlet hierarchy construction, on the top of the Halperin (zeroth-level) state we have the spin-singlet state of quasiparticles.

The effects of the statistics in Eq. (3.45) are in the product with absolute differences, and, as we know from before, if the densities or total numbers of the (quasi)particles that characterize the ground state are concerned, they are irrelevant [26]. (That can be showed by an application of the Laughlin plasma analogy [1] to the wave function.) If we consider the quasiparticles as fermionic and, loosely speaking, pull the factor $\prod_{i<j}\left(w_{i \uparrow}-w_{j \uparrow}\right) \prod_{i<j}\left(w_{i \downarrow}-w_{j \downarrow}\right)$ from the product with the absolute differences in Eq. (3.45) to the product (under the integral signs) involving the differences of $z$ 's and $w$ 's, and the factor $\prod_{i<j}\left(\bar{w}_{i \uparrow}-\bar{w}_{j \uparrow}\right) \prod_{i<j}\left(\bar{w}_{i \downarrow}-\bar{w}_{j \downarrow}\right)$ to the product with the differences of $\bar{w}$ 's to the $2 n^{t h}$ power, effectively the state of quasiparticles will be a Halperin spin-singlet state. So in this case, we have also a spin-singlet state on the top of another one. This is to be expected, because the quasiparticles are taken to be fermionic in the lowest Landau level just because of their way of combining together with respect to the internal spin degree of freedom [7].

In the second case, with $D_{s}^{2}$ in (3.38), the spin-singlet requirement is $m_{1}-n_{1}=2$. The matrix $D_{c}^{2}$ is the same as in the previous construction. When $n_{1}=0$ i.e. $m_{1}=2$ (and $m_{0}$ and $n_{0}$ are of the Halperin states) $D_{s}^{2}$ and $D_{c}^{2}$ describe a simple Jain's construction: two Landau levels are completely filled with composite fermions obtained by attaching $\left(m_{0}+n_{0}-1\right) / 2$ flux quanta to electrons. In the standard-hierarchy picture this is a quasielectron construction. The filling fraction is

$$
\nu=\frac{4}{4\left[\frac{m_{0}+n_{0}-1}{2}\right]+1} .
$$

\section{Arbitrary-level spin-singlet hierarchy}

The starting point for the $n^{\text {th }}$-level-hierarchy construction is the spin matrix $\Lambda_{s}$ (in the standard-hierarchy basis):

$$
\Lambda_{s}^{n}=\left(\begin{array}{cccccc}
m_{0}-n_{0} & 1 & 0 & \cdots & 0 & 0 \\
1 & m_{1}-n_{1} & 1 & \cdots & 0 & 0 \\
0 & 1 & m_{2}-n_{2} & \cdots & 0 & 0 \\
\vdots & \vdots & \vdots & \ddots & 1 & 0 \\
0 & 0 & 0 & 1 & m_{n-1}-n_{n-1} & 1 \\
0 & 0 & 0 & 0 & 1 & m_{n}-n_{n}
\end{array}\right)
$$

The spin-singlet condition, formulated in the previous subsection and easily generalized to any level of the hierarchy, demands that the diagonal entries are such that $\left|\operatorname{det} \Lambda_{s}\right|=1$. We satisfy this condition by demanding that, at each level $i$ of the hierarchy, $\left|\operatorname{det} \Lambda_{s}^{i}\right|=1$. This means that the differences $m_{i}-n_{i}, i=0, \ldots, n$, become fixed numbers characterizing the constructions. To determine possibilities we define matrices $M_{k}, k=0, \ldots, n$, of the form 


$$
M_{k}=\left(\begin{array}{ccccc}
m_{k}-n_{k} & 1 & 0 & \cdots & 0 \\
1 & m_{k+1}-n_{k+1} & 1 & \cdots & 0 \\
0 & 1 & m_{k+2}-n_{k+2} & \cdots & 0 \\
\vdots & \vdots & \vdots & \ddots & 1 \\
0 & 0 & 0 & 1 & m_{n}-n_{n}
\end{array}\right)
$$

They satisfy the following recursion relation:

$$
\operatorname{det} M_{k}=\left(m_{k}-n_{k}\right) \operatorname{det} M_{k+1}-\operatorname{det} M_{k+2},
$$

where $\operatorname{det} M_{n+1}=1$ and $\operatorname{det} M_{n+2}=0$. Repeatedly requiring at each level $i$ of the hierarchy that $\left|\operatorname{det} \Lambda_{s}^{i}\right|=1$, we have, at the end, this constraint on the values of $m_{n}$ and $n_{n}$ :

$$
\left|\operatorname{det} \Lambda_{s}^{n}\right|=\left|\operatorname{det} M_{n} \pm \operatorname{det} M_{n+1}\right|=\left|\left(m_{n}-n_{n}\right) \pm 1\right|=1 \text {, }
$$

where the sign depends on the way the lower level was constructed.

Because of the nominal Bose statistics of the quasiparticles in this field theory construction $\left(m_{n}\right.$ is even), and as a consequence of the earlier requirement, $m_{n}+n_{n}$ is an even number. The filling fraction in this construction is given by

$$
\nu_{n}=2\left(\Lambda_{c}^{-1}\right)_{00}=2 \frac{1}{m_{0}+n_{0}-\frac{1}{m_{1}+n_{1}-} \cdot{ }_{-\frac{1}{m_{n}+n_{n}}}} .
$$

Because $m_{0}+n_{0}$ is an odd number and all the rest are even, the general form of the filling fraction is an even over an odd integer.

At each level of the hierarchy we may constrain the signs of $m_{n}$ and $n_{n}$, demanding that, in the basis of Jain's construction we begin (combining one Landau level) with a Halperin state. This, as we did in the case of the first-level quasihole construction, is not necessary. The Chern-Simons field theory, that we use, enlarges the principles of the standard-hierarchy construction ( [2,7]), and contains also constructions where ground states of the (quasi)particle systems can be described by wave functions which are not of the form of the Halperin states. Namely, if we take the function of the filled lowest Landau level of both spins and attach an even number $(2 n)$ of flux quanta, in the direction opposite to the external magnetic field, we get a spin-singlet state, at the filling fraction $\nu^{-1}=(4 n-1) / 2$, which differs from the Halperin states. These wave functions, projected to the lowest Landau level, are included in Jain's constructions. As a consequence, this enlarged construction, (with no additional constraints on $m_{n}$ 's and $n_{n}$ 's), covers all filling fractions of the even integer/odd integer form.

By induction, the matrix $S_{n}$, at each level $n$, can be found, which diagonalizes the matrix $\Lambda_{s}^{n}$ and gives the corresponding charge matrix in the basis of Jain's construction. We assume the form of $S_{n}^{\top}$, a $(n+1) \times(n+1)$ matrix, to be 


$$
S_{n}^{\top}=\left(\begin{array}{cccccc}
1 & 0 & 0 & 0 & \cdots & 0 \\
1 & -1 & 0 & 0 & \cdots & 0 \\
1 & -1 & (-1)^{k_{2}} & 0 & \cdots & 0 \\
1 & -1 & (-1)^{k_{2}} & (-1)^{k_{3}} & \cdots & 0 \\
\vdots & \vdots & \vdots & \vdots & \ddots & \vdots \\
1 & -1 & (-1)^{k_{2}} & (-1)^{k_{3}} & \cdots & z
\end{array}\right)
$$

where each $k_{i}, i=2, \ldots, n-1$, can be 0 or 1 . $S_{n-1}^{\top}$, a $n \times n$ matrix, lies in the upper left corner of $S_{n}^{\top}$. If, for some choice of $\left(k_{2}, \ldots, k_{n-1}\right), S_{n-1}^{\top}$ diagonalizes given $\Lambda_{s}^{n-1}$ matrix for the $(n-1)^{\text {th }}$ level of the hierarchy (so that the diagonal entries are +1 and -1 ), and $S_{n}^{\top}$ diagonalizes in the same way $\Lambda_{s}^{n}$, we will show that $|z|=1$ and the sign of $z$ is uniquely determined by the way the lower level is constructed. From the form of $S_{n}^{\top},(3.55)$, and the previous assumptions follows that

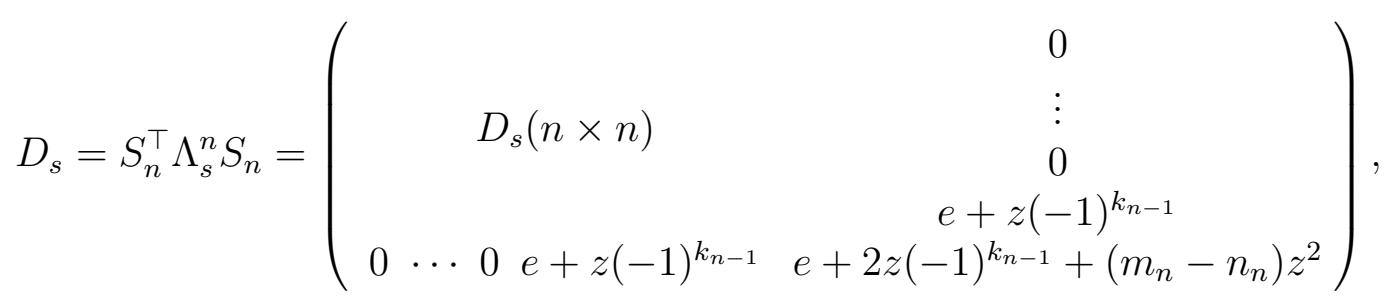

where $D_{s}(n \times n)$ is a $n \times n$ diagonal matrix with all diagonal entries +1 or -1 , and $e$ represents its last diagonal entry. From the constraint that the off-diagonal elements of $D_{s}$ are also zero follows that $z=(-1)^{k_{n-1}+1}$ e (i.e. $\left.|z|=1\right)$.

\section{GENERALIZED SPIN-SINGLET HIERARCHY}

We may consider also constructions for ground states of spin-singlet FQHE systems where, at some stage of the hierarchy, elementary quasiparticles of a Halperin state pair into spinless Laughlin quasiparticles and make a Laughlin state. This situation, in our formalism, in the case of the first-level spin-singlet hierarchy, is described by the following matrix for the charge degrees of freedom:

$$
\Lambda_{c}=\left(\begin{array}{cc}
m_{0}+n_{0} & 2 \\
2 & 4 p
\end{array}\right),
$$

where $m_{0}$ and $n_{0}$ are of the Halperin zeroth level state, and $p$ is an arbitrary integer, and matrix

$$
\Lambda_{s}=\left(\begin{array}{ll}
1 & 0 \\
0 & 0
\end{array}\right)
$$

for the spin degrees of freedom. (The factor 2 in the charge matrix, for the part that describes the new construction, which is similar to the standard-hierarchy construction in the polarized case, is again a consequence of the choice for the unit of flux in our formalism.) The zero on the diagonal of $\Lambda_{s}$ is a consequence of the fact that the description of the localized quasiparticle excitations, in this system, can be given by a three-dimensional lattice. 
Again, by applying the matrix transformation $S$ on both spin and charge part simultaneously we may find different expressions of the same construction. In the special case when $S$ is given by (3.39), the charge and spin matrix are

$$
J_{c}=\left(\begin{array}{cc}
4 n+1 & 4 n-1 \\
4 n-1 & 4 n-3+4 p
\end{array}\right) \quad \text { and } \quad J_{s}=\left(\begin{array}{ll}
1 & 1 \\
1 & 1
\end{array}\right),
$$

where $m_{0}+n_{0}=4 n+1$. When $n=1$ (i.e. in the previous hierarchy construction we begin with the $\nu=2 / 5$ Halperin state) and $p=1$, the matrices describe the $\nu=1 / 2$ spin-singlet state of the following Jain's construction:

$$
\Psi_{\nu=\frac{1}{2}}=\chi_{2} \chi_{1} \chi_{1,1} .
$$

$\chi_{i}, i=1,2$, denote in Jain's notation [4] a filled lowest Landau level and filled first two Landau levels without regard to the spin degree of freedom, and $\chi_{1,1}$ denotes a lowest Landau level occupied with particles of both spins. Explicitly, the flux attaching factor, $\chi_{1} \chi_{1,1}$, can be rewritten as

$$
\prod_{i<j}\left(z_{i}-z_{j}\right)^{\frac{3}{2}} \times \prod_{m<n}\left(z_{m \uparrow}-z_{n \uparrow}\right)^{\frac{1}{2}} \prod_{k<l}\left(z_{k \downarrow}-z_{l \downarrow}\right)^{\frac{1}{2}} \prod_{r, s}\left(z_{r \uparrow}-z_{s \downarrow}\right)^{-\frac{1}{2}}
$$

which means that $\frac{3}{2}$ flux quanta are attached in the usual way, i.e.

$$
J_{c}(n=1, p=1)=\left(\begin{array}{ll}
2 & 0 \\
0 & 2
\end{array}\right)+3\left(\begin{array}{ll}
1 & 1 \\
1 & 1
\end{array}\right),
$$

and there is also the flux attaching (without net flux attached) in the spin part described by $J_{s}$ in (4.3).

By the way of the gluing theory, as in Sec. III A, we may find out, in this special case, that the glue vectors are

$$
\left(\begin{array}{l}
0 \\
0
\end{array}\right)_{c} \oplus(0)_{s} \text { and }\left(\begin{array}{l}
5 \\
2
\end{array}\right)_{c} \oplus(1)_{s} .
$$

The charge part is given in the basis of the corresponding charge excitation lattice $\left(L_{c}^{*}\right)$ in which the Gram matrix is given by $(1 / 2) \Lambda_{c}^{-1}$ (see Eq.(4.1)) with $m_{0}+n_{0}=5$ and $p=1$. We used one-component vectors, in the spin part, associated with the single direction that describes the spin of the localized excitations. For a basis of the order-parameter lattice that is obtained by the way of the gluing we can choose vectors:

$$
\left(\begin{array}{l}
4 \\
8
\end{array}\right)_{c} \oplus(0)_{s},\left(\begin{array}{l}
5 \\
2
\end{array}\right)_{c} \oplus(1)_{s} \text {, and }\left(\begin{array}{l}
0 \\
0
\end{array}\right)_{c} \oplus(2)_{s}
$$

(with the first and last vector describing a pure charge and pure spin order-parameter excitation, respectively). The corresponding three-dimensional Gram matrix is

$$
\left(\begin{array}{lll}
4 & 2 & 0 \\
2 & 3 & 1 \\
0 & 1 & 2
\end{array}\right) .
$$

It is straightforward to obtain higher level generalizations of this construction and we will only remark that, in Jain's picture, they are always followed by the flux attaching procedure to the spin degrees of freedom described previously. 


\section{PARTIALLY POLARIZED HIERARCHY}

At some level of the spin-singlet hierarchy we may allow only same-spin quasiparticles to make a Laughlin state [8]. The equations that we can easily write down to describe this configuration are characterized by the following charge matrix in the case of the first level hierarchy:

$$
\Lambda_{c}=\left(\begin{array}{cc}
m_{0}+n_{0} & 1 \\
1 & 4 p-1
\end{array}\right),
$$

where $p$ is an arbitrary integer and, as before, $m_{0}$ and $n_{0}$ are of the Halperin zeroth-level state. Only in this case, i.e. when $m_{0}-n_{0}=1$, our charge matrix is an integer matrix and the invariance under the integer matrix $S$ can be considered. If $S$ is given by (3.39), the transformed $\Lambda_{c}$ is

$$
J_{c}=\left(\begin{array}{cc}
m_{0}+n_{0} & m_{0}+n_{0}-1 \\
m_{0}+n_{0}-1 & m_{0}+n_{0}-1+4 p-2
\end{array}\right),
$$

i.e. we are again in the basis of Jain's construction for partially polarized states [4]. When $m_{0}+n_{0}=1$ and $p=1$, the matrix also describes the simplest example of partially polarized states with the lowest Landau level filled with both spins and the second level (partially) filled with only one spin projection.

It is important to point out that, even in the cases of the higher level constructions, the charge matrix is an integer matrix if $m_{i}^{\prime} \mathrm{s}$ and $n_{i}{ }^{\prime} \mathrm{s}$ of the levels with no net spin polarization are chosen to describe a spin-singlet construction. Therefore, the presence of the $\mathrm{SU}(2)$ symmetry (which is broken in the ground state) is followed by the possibility to describe the invariance of the system under change of the hierarchy basis in the picture which treats the charge and spin degrees of freedom separately. Then the gluing theory description, with the integral order-parameter charge and spin lattices is appropriate.

\section{CONCLUSION}

In conclusion, the invariance of FQHE systems with spin under change of the hierarchy basis can be also described in the spin-charge decomposition picture. We characterized the integral charge and spin lattices of the order parameters of the systems by stating the conditions that the spin-lattice Gram matrix should satisfy to describe SU(2)-invariant constructions. In particular, for spin-singlet constructions, a basis must exist in which the Gram matrix is diagonal with +2 or -2 on the diagonal. This is a cosequence of the existence of as many $\mathrm{SU}(2)$ symmetries as there are levels in a specific spin-singlet construction. The physical spin, i.e. spin independent of the levels of the hierarchy, in this basis, is a sum of the spin quantum numbers connected with each $\mathrm{SU}(2)$ symmetry. The excitation lattices of all these systems are described as lattices of the gluing theory with spin only and charge only excitations glued in a special way. The formalism was extended to the generalized spin-singlet states and partially polarized states.

The lattice approach, which we used, is a systematic way to classify all abelian FQHE states with spin. We attempted to include all states proposed earlier in the literature, 
in a description convenient for future applications. The standard-hierarchy spin-singlet construction that we proposed, in Sec. III B, disagrees with the construction in Ref. [8]. If the nominal statistics of quasiparticles is taken to be fermionic, like in Ref. [8], the algebraic equations of the construction are still the same ones as in (3.2) with $m_{1}$ even (as in (3.2)). This, although not obvious at first sight, can be seen by looking at the wave function (of a specific spin-singlet construction) in (3.45), and reading the comments in the paragraph just following. (The fermionic construction requires extra, flux-attaching factors (with respect to the bosonic one), in the lowest Landau level, to be complete.) More importantly, our construction is justified by a clear-cut, spin-singlet condition in the same subsection.

The experimental findings around filling fraction 3/2 in Ref. [12] are in agreement with our conclusion that, if we do not consider generalized constructions, the spin-singlet states can occur only at the filling fractions of the form even over odd integer. Their results are consistent with the composite-fermion descrption of partially polarized states [ 4 . Once we have the lattice description, presented here, we may consider the "stability" of various constructions, i.e. likelihood that they correspond to the systems with well developed plateaus in experiments [29]. It is interesting in this respect to note that the lattices corresponding to the states described in Ref. [12] have positive-definite Gram matrices.

Research was supported by NSF Grant No. DMR-91-57484. 


\section{REFERENCES}

[1] R.B. Laughlin, Phys. Rev. Lett. 50, 1395 (1983).

[2] F.D.M. Haldane, Phys. Rev. Lett. 51, 645 (1983).

[3] B.I. Halperin, Phys. Rev. Lett. 52, 1583 (1984).

[4] J.K. Jain, Phys. Rev. Lett. 63, 199 (1989); Phys. Rev. B 40, 8079 (1989); 41, 7653 (1990).

[5] N. Read, Phys. Rev. Lett. 65, 1502 (1990).

[6] B.I. Halperin, Helv. Phys. Acta 65, 75 (1983).

[7] F.D.M. Haldane in The Quantum Hall Effect, edited by R.E. Prange and S.M. Girvin (Second edition, Springer Verlag, New York, 1990).

[8] E.H. Rezayi, Phys. Rev. B 3913541 (1989).

[9] D.H. Lee and C.L. Kane, Phys. Rev. Lett. 64, 1313 (1990).

[10] G. Moore and N. Read, Nucl. Phys. B360, 362 (1991).

[11] A. Lopez and E. Fradkin, Phys. Rev. B 51, 4347 (1995).

[12] R.R. Du, A.S. Yeh, H.L. Stormer, D.C. Tsui, L.N. Pfeifer, and K.W. West, Phys. Rev. Lett. 75, 3926 (1995).

[13] C. Nayak and F. Wilczek, Nucl. Phys. B450, 558 (1995).

[14] J.H. Conway and N.J.A. Sloane, Sphere Packings, Lattices and Groups (Springer Verlag, New York, 1988).

[15] S.-C. Zhang, H. Hansson, and S. Kivelson, Phys. Rev. Lett. 62, 82 (1989).

[16] Z.F. Ezawa and A. Iwazaki, Phys. Rev. B 47, 7295 (1993).

[17] K. Moon, H. Mori, Kun Yang, S.M. Girvin, A.H. MacDonald, L. Zheng, D. Yoshioka, and S.-C. Zhang, Phys. Rev. B 51, 5138 (1995).

[18] M. Milovanović and N. Read, Phys. Rev. B 53, 13559 (1996).

[19] S.-C. Zhang, Int. J. Mod. Phys. B6, 25 (1992).

[20] R. Jackiw in Current Algebra and Anomalies, (Princeton University Press, New Jersey, 1985).

[21] E. Witten, Comm. Math. Phys. 121, 351 (1989).

[22] A. Balatsky and E. Fradkin, Phys. Rev. B 43, 10622 (1991) and references therein.

[23] B. Blok and X.-G. Wen, Phys. Rev. B 42, 8145 (1990).

[24] S.M. Girvin and A.H. MacDonald, Phys. Rev. Lett. 58, 1252 (1987).

[25] X.-G. Wen and A. Zee, Phys. Rev. B 46, 2290 (1992).

[26] B. Blok and X.-G. Wen, Phys. Rev. B 43, 8337 (1991).

[27] B.I. Halperin, Phys. Rev. Lett. 52, 1583 (1984), R.B. Laughlin in The Quantum Hall Effect, edited by R.E. Prange and S.M. Girvin (Second edition, Springer Verlag, New York,1990), B. Blok and X.-G. Wen, Phys. Rev. B 43, 8337 (1991).

[28] M. Hammermesh, Group Theory and its Application to Physical Problems, (Dover, New York, 1989).

[29] F.D.M. Haldane, Phys. Rev. Lett. 74, 2090 (1995). 